\title{
Interfacial Behavior of Acetalized Poly(vinyl alcohol) Monolayers Observed by Brewster Angle Microscopy and Canal Surface Viscometry
}

\author{
Nobuhiro SAто, Shinzaburo Ito, and Masahide Yamamoto ${ }^{\dagger}$ \\ Department of Polymer Chemistry, Graduate School of Engineering, Kyoto University, \\ Sakyo-ku, Kyoto 606-01, Japan
}

(Received February 26, 1996)

\begin{abstract}
The interfacial properties of polymer monolayers at the air/water interface has been determined by the observation of morphological changes and the measurement of surface shear viscosity. Brewster angle microscopy (BAM) was applied to the monolayers of the acetalized poly(vinyl alcohol)s having alkyl side chains of various lengths. The monolayers of these polymers form coherent domains even at a large surface area, and the longer the side chains are, the smaller the degree of domain deformation is. Canal surface viscometry also showed that shear viscosity of the monolayer increases with the increase in the number of side-chain carbons. These findings indicate that the cohesive force is stronger among the side chains protruding out of the interface when the alkyl chains are longer, so that the entire monolayer becomes more viscous while retaining the liquid-like character.

KEY WORDS Surface Viscosity / Acetalized Poly(vinyl alcohol) / Polymer Monolayer / Brewster Angle Microscopy / Canal Surface Viscometer/
\end{abstract}

Some amphiphilic polymers such as poly(methyl methacrylate) and poly(vinyl acetate) form monomolecular films upon a clean surface of water. ${ }^{1,2}$ The particular behavior of the film-forming polymers confined in the quasi 2-dimensional plane is an interesting subject of polymer science as well as interface science. ${ }^{3-5}$ For preparing functional devices, polymer monolayers have many advantages in thermal and mechanical stability, uniform dispersion of the functional groups, and thinness of each layer compared with low-molecular-weight monolayers. ${ }^{6-8}$ However, we have little information about the structure, dynamics and other fundamental properties of the polymer monolayers at the air/water interface.

In the 1940s Crisp ${ }^{9,10}$ investigated many kinds of polymers and classified them systematically according to the stability of the monolayer from the measurements of surface pressure-area isotherms and surface potential. Although his work has been the basis of the present studies, the mechanical properties such as elasticity, viscosity and viscoelasticity, which are remarkable features of polymer monolayers, has not been investigated sufficiently. These properties are of crucial importance to the quality of Langmuir-Blodgett (LB) films which are fabricated by the successive deposition of monolayers onto a solid substrate, because the deposition process is much affected by the mechanical properties of the monolayers on the water surface. In addition to such applicative interests, it is worthwhile from a scientific point of view to study the interfacial properties of the monolayer in relation to structure of polymers at the molecular level. ${ }^{5,11}$

Herein, we mainly studied the surface shear viscosity of acetalized PVAs which have good transferability onto various solid substrates. ${ }^{12}$ To clarify the relationship between the viscosity and the molecular structure of the polymers, we employed Brewster angle microscopy (BAM) and canal surface viscometry; the former displays the morphological structure of the monolayer, the latter gives the quantitative value of the surface viscosity.
BAM enables us to visualize the form of monolayers in situ without any additives like fluorescent probes and without the deposition onto a solid substrate. ${ }^{13,14}$ For this reason, BAM is suitable for inspecting the behavior of the monolayer on the water surface. Canal surface viscometry, on the other hand, is a simple method for measuring surface shear viscosity. The relation of the side-chain length of the polymers to the morphology and surface viscosity of their monolayers is demonstrated by using these methods.

\section{EXPERIMENTAL}

\section{Materials}

Poly(vinyl alkanal acetal)s were synthesized by the acetalization of two kinds of PVAs (Wako Pure Chemical, $D P=2000$; Aldrich, $M W=13000-23000$ ) with linear alkyl aldehydes (Figure 1). The detailed synthetic procedure was given elsewhere. ${ }^{15}$ For the purification, obtained polymers were precipitated from a benzene solution into methanol three times, except PVB, which was poured into hexane from an acetone solution. The fraction of alkyl units represented by $x$ in Figure 1 was determined by the elementary analysis (Table I). The high-molecular-weight samples $(D P=2000)$ were used for the BAM observation. On the other hand, the lowmolecular-weight samples $(D P=1000)$ were used for the
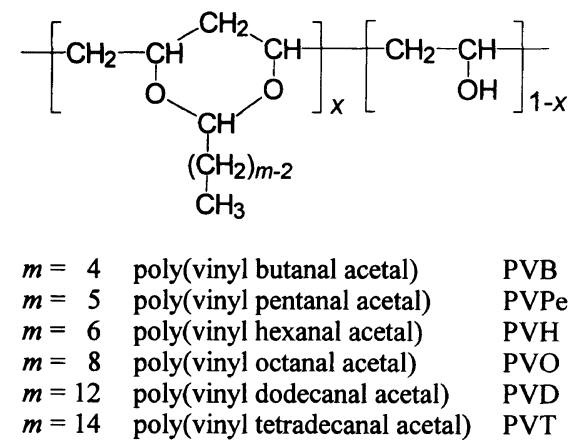

Figure 1. Molecular structure of sample polymers. 
Table I. Mole fraction ( $x$ ) of alkyl unit in each sample

\begin{tabular}{cccc}
\hline$m$ & Sample & $D P$ of PVA & $x$ \\
\hline 4 & PVB & $1000^{\mathrm{a}}$ & 0.77 \\
5 & PVPe & 2000 & 0.70 \\
6 & PVH & $1000^{\mathrm{a}}$ & 0.73 \\
8 & PVO & $1000^{\mathrm{a}}$ & 0.73 \\
& & 2000 & 0.69 \\
12 & PVD & $1000^{\mathrm{a}}$ & 0.72 \\
& & 2000 & 0.66 \\
14 & PVT & $1000^{\mathrm{a}}$ & 0.79 \\
& & 2000 & 0.70 \\
\hline
\end{tabular}

a $\quad M W=13000-23000$ according to the manufacturer. However, our light scattering measurement yielded $M W=48000$, which corresponds to $D P=1000$.

viscosity measurement, because the surface viscosity of the high-molecular-weight samples was too high for our viscometer to yield accurate results.

Poly(octadecyl methacrylate) (PODMA) was synthesized by the radical polymerization with azobisisobutyronitrile as an initiator. The weight-average molecular weight was about 50000 determined by GPC with reference to standard polystyrene.

\section{Monolayer Preparation}

Monolayers were prepared on the surface of pure water, which was ion-exchanged, distilled and then treated with a water purification system (Barnstead NANO PURE II). A benzene solution (Dojin Spectrograde) of the polymer ( $c a .0 .1 \mathrm{~g} \mathrm{dm}^{-3}$ ) was dropped onto the water surface with a microsyringe. All measurements and observations were carried out at $20 \mathrm{~min}$ after the spread to achieve the equilibrium of the monolayer and the complete evaporation of solvent. A Teflon-coated aluminum trough (Kenkosha model SI-2; $600 \mathrm{~mm} \times$ $150 \mathrm{~mm} \times 7.5 \mathrm{~mm}$ ) was equipped with Teflon barriers to change the surface area. A water circulation system was installed under the trough for temperature control with the precision of $0.1{ }^{\circ} \mathrm{C}$. The room temperature was also controlled with an air conditioner to as close to water temperature as possible. Surface pressure was measured with a Wilhelmy plate of sandblasted glass and an electronic balance (A \& D ER-60A).

\section{Brewster Angle Microscopy}

A homemade Brewster angle microscope was set up as shown in Figure 2. $P$-Polarized light of a $10 \mathrm{~mW}$ He-Ne laser (Uniphase model $1135 \mathrm{P}$ ) at $\lambda=632.8 \mathrm{~nm}$ through a polarizer (Glan-Thompson prism, Sigma Koki) was impinged upon the water surface. The angle of incidence was set to be $53.1^{\circ}$ which is the Brewster angle for the air/water interface. Practically we adopted the angle at which the reflected light gave minimum intensity. An optical filter was placed under the observed area to suppress the reflection of light transmitted through the interface. The optical image was magnified with objective lenses or a singlet lens $(f=50 \mathrm{~mm})$ and introduced to a CCD camera (Hitachi electronics) equipped with a camera controller (Hamamatsu C2400). All observation was carried out at $20.0^{\circ} \mathrm{C}$.

\section{Surface Viscometry}

We employed a canal surface viscometer made of

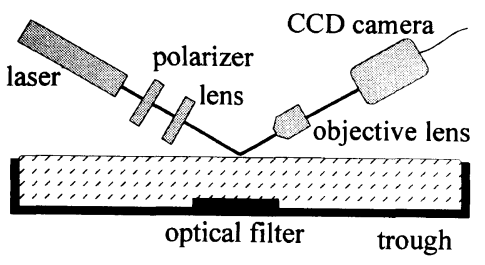

Figure 2. Brewster angle microscopy setup.

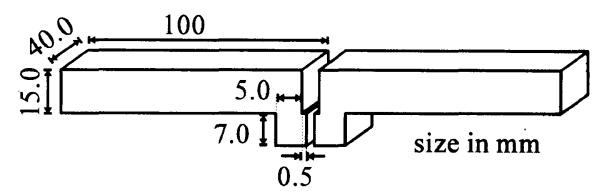

Figure 3. Dimension of the canal surface viscometer.

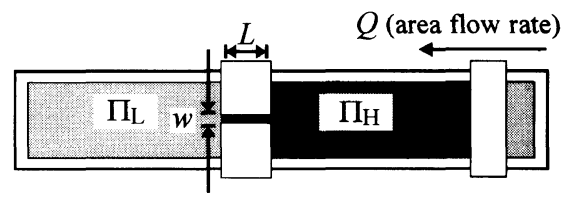

Figure 4. Schematic representation of the canal surface viscometer.

Teflon as shown in Figure 3. Sacchetti et al. reported that this type of the canal viscometer gave reproducible data. ${ }^{16}$ The canal length $L$ was $40 \mathrm{~mm}$ and the canal width $w$ ranged from 0.5 to $3 \mathrm{~mm}$. A small step was made on the canal wall to prevent a concave-down meniscus of water.

At the beginning a Teflon barrier was placed on one side of the canal to prevent the surface flow. The monolayer was prepared on the other side of the canal, and 20 min later compressed after the evaporation of the solvent. Keeping the surface pressure to a fixed value $\left(\Pi_{\mathrm{H}}\right)$ with a computer-controlled feedback system, the former barrier was rapidly slid away and enough space made for the monolayer to flow in freely. Since the surface pressure of the free surface is considered to be zero under such conditions, the difference of the surface pressure $\Delta \Pi$ between the two sides of the canal approximately equals $\Pi_{\mathrm{H}}$ during the measurement. Surface viscosity $\eta_{\mathrm{s}}$ of the monolayer is calculated from the area flow rate $Q$ by the following equation.

$$
\eta_{\mathrm{s}}=\frac{\Delta \Pi w^{3}}{12 L Q}-\frac{w \eta_{0}}{\pi}
$$

where $\eta_{0}$ is the bulk viscosity of subphase. Schematic illustration of the measurement is shown in Figure 4.

All measurements were carried out at $20.0^{\circ} \mathrm{C}$ with $\Pi_{\mathrm{H}}=2 \mathrm{mN} \mathrm{m}^{-1}$.

\section{RESULTS AND DISCUSSION}

\section{Surface Pressure-Area Isotherms}

Figure 5 shows the surface pressure-area $(\Pi-A)$ isotherm of each sample measured at $20.0^{\circ} \mathrm{C}$. As the number of side-chain carbons increased, the compression of the monolayer gave rise to the steeper increase of the surface pressure and the pressure in the plateau region of the isotherm reached a higher value (except PVT), indicating that the longer side chains result in a more condensed monolayer. The plateau region of the isotherms can be ascribed to the collapse of the mono- 


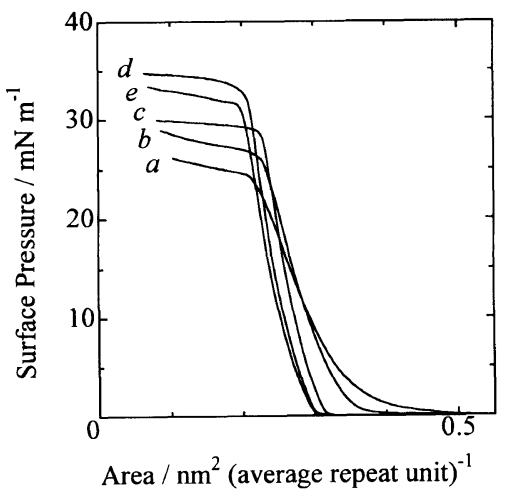

Figure 5. $\Pi-A$ isotherms of the acetalized PVAs having side chains with different lengths. The samples of $D P=1000$ were used for the measurement. (a) PVB; (b) PVH; (c) PVO; (d) PVD; (e) PVT.

layer and the experimental evidence of this is shown in the next subsection. The reason why the plateau pressure of the PVT monolayer was lower than that of the PVD monolayer is still unclear, but one possible explanation is that the impurity of the aldehydes used for the acetalization may caused the anomalous plateau pressure of the PVT monolayer.

\section{Morphology of Acetalized PVA Monolayers}

The observation by Brewster angle microscopy revealed the following features of the morphology of the acetalized PVA monolayers. Figure 6 is a typical BAM image of a PVD $(m=12)$ monolayer. At a larger surface area, it formed the so-called island structure, and $2-3$ mm domains having smoothly curved boundaries drifted upon the water surface. The domains easily underwent deformation by the surface flow of the water, showing the soft and liquid-like properties; consequently various domain shapes with round but deformed features were seen under the scope. The intensity of the light reflected from the domain was uniform, which implies that there was no distinctive alteration of orientation or structure within the domain. A similar morphology was also observed for all of the acetalized PVAs used. These morphological features were in contrast to the monolayer of poly(octadecyl methacrylate). This polymer formed a rigid and angular domain, the reflectivity of which was not uniform (Figure 7). Such solid-like behavior of PODMA is explained by the crystallization of the side chains.

On the course of compression and expansion of the water surface, one can see the coalescence and the separation of the domains of the PVD monolayer. Figure 8a shows that the unified domain appeared seamless and uniform, while the separating domain showed ductile deformation at the dividing part as seen in Figure $8 \mathrm{~b}$.

As compressed up to the plateau region of the isotherm, a wrinkle-like structure perpendicular to the compressing direction appeared in the domain (Figure 9). These wrinkles obviously indicates the collapse of the monolayer, that is, transformation to the multi-layer or the 3-dimensional bulk structure.

Judging from the above findings, the monolayers of acetalized PVAs behave like a 2-dimensional melt or very viscous liquid on the water surface. This amorphous and fluid character is accounted for by the several reasons

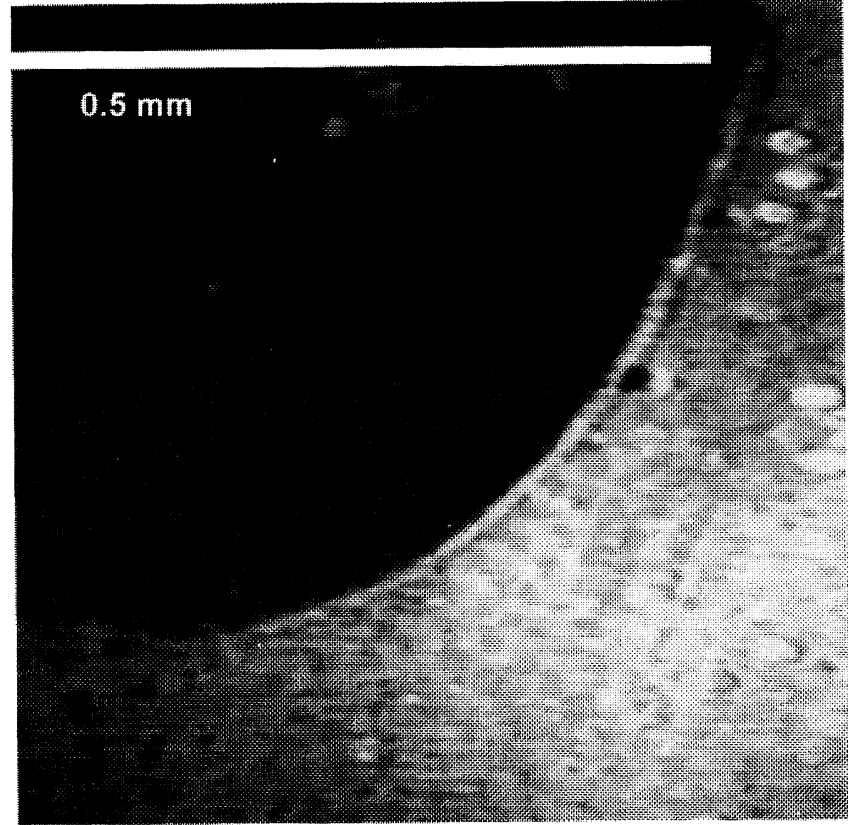

Figure 6. Morphology of a PVD monolayer observed at a surface pressure of $0 \mathrm{mN} \mathrm{m}^{-1}$. The bright area corresponds to the monolayer and the dark area to the water surface. Interference of laser light caused the image striped. The center part of the image was brighter than the periphery owing to the distribution of the incident laser light intensity.

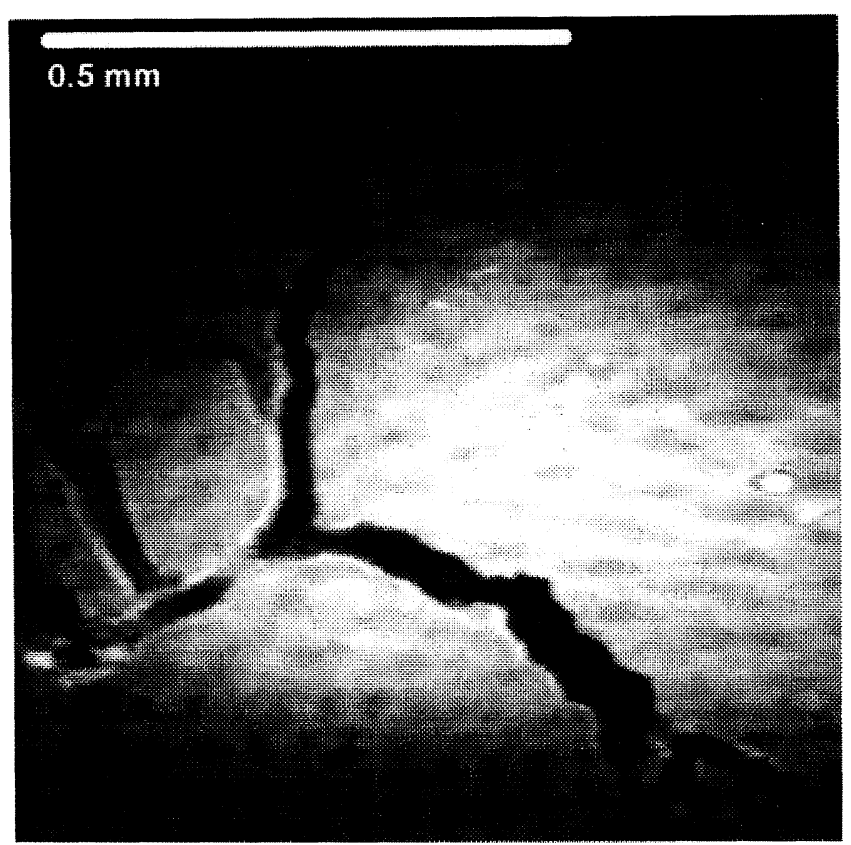

Figure 7. Angular domains of $\operatorname{PODMA}\left(\Pi=0 \mathrm{mN} \mathrm{m}^{-1}\right)$.

based on the molecular structure of the polymers. Firstly, the side chains are not long enough to cause their crystallization. Secondly, the hydrophobic side chains cannot be packed closely together because the side chains are attached to the bulky acetal-ring which has a larger area than the cross section of the alkyl chain. Finally, the segments having unreacted hydroxyl groups provide the flexibility to the backbone of the polymers because the segments with hydroxyl groups have no hydrophobic alkyl chains which hinder the free rotation of the covalent bond of the backbone.

The degree of domain deformation was dependent on the side-chain length of the polymer. PVPe $(m=5)$ and 

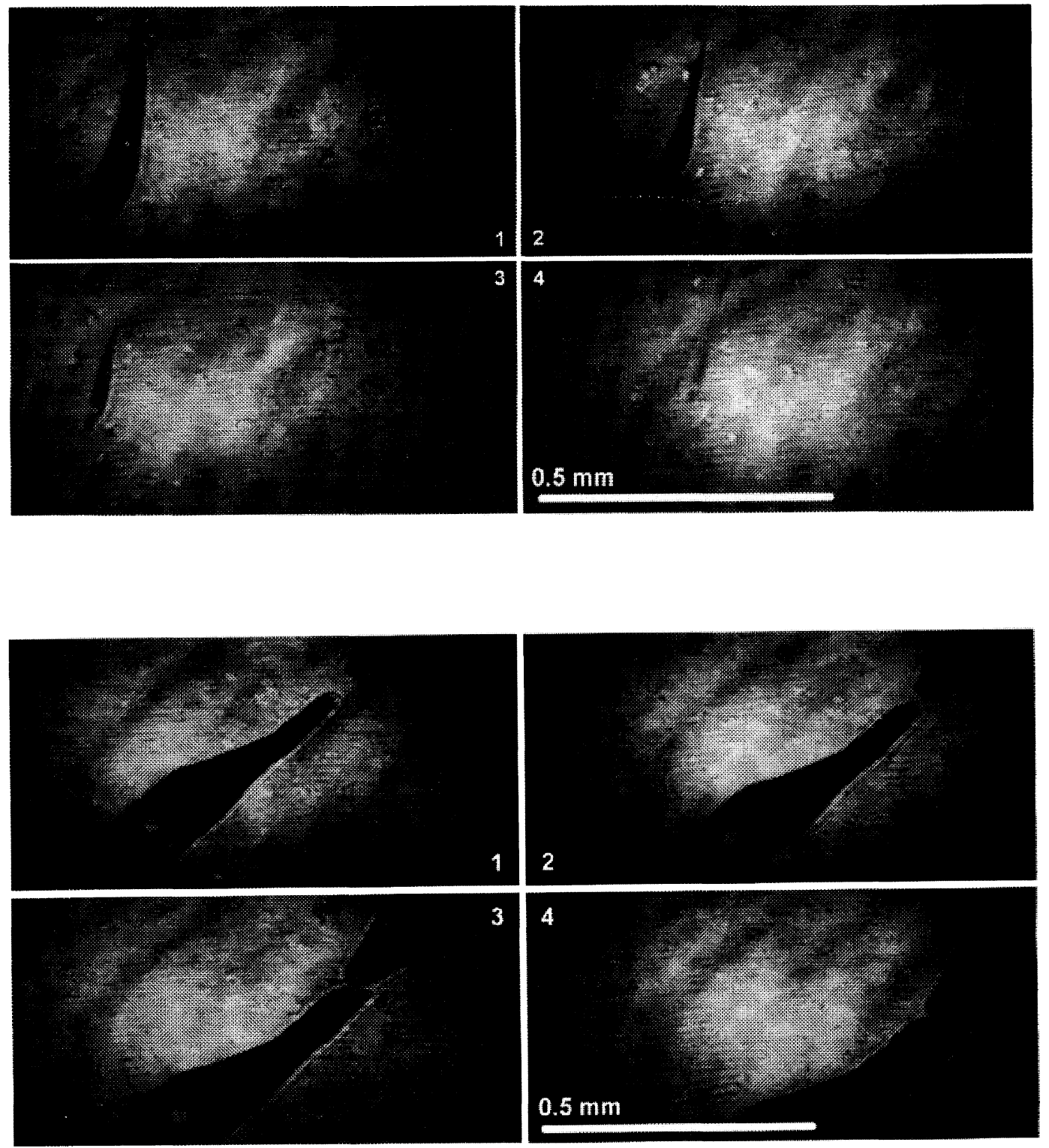

Figure 8. Top: (a) Coalescence of two domains of a PVD monolayer. A seamless and uniform domain was formed. These pictures (1-4) were taken at every $5 \mathrm{~s}$ intervals. Bottom: (b) Separation of a domain of a PVD monolayer. The dividing part was extended. These were taken at every $1 \mathrm{~s}$ intervals. Both images were taken at $\Pi=0 \mathrm{mN} \mathrm{m}^{-1}$.

PVO $(m=8)$, which possess shorter side chains, formed stripe domains as shown in Figure 10, whereas this characteristic shape was not seen for the PVD and PVT monolayers.

This difference was more distinctive in the following experiment. Figure 11 shows the morphology of the domains passed through the $1 \mathrm{~mm}$ wide canal placed on the water surface when $2 \mathrm{mN} \mathrm{m}^{-1}$ surface pressure was applied to one side of the canal. The flowed-out domains of PVT took a ribbon-like shape (Figure 11a), while PVO domains showed an indefinite shape (Figure 11b), although both PVO and PVT domains were considered to be a ribbon-like shape immediately after passage through the canal. These are visible indications of the viscous feature of the monolayers under the shear stress, that is, the monolayers of polymers with shorter side chains (PVPe, PVO) were so deformable by the surface flow of water that they could not keep their shape. Thus, BAM revealed that the monolayer viscosity is strongly affected by the side-chain length of the polymer.

\section{Viscosity of the Monolayers}

The canal surface viscometry provided quantitative evidence of a relationship between the side-chain length and the viscosity of the monolayer (Figure 12). The surface viscosity exponentially increased as a function of the side-chain carbon number. Unfortunately, the experimental error was rather large probably owing to the uncertainty of the canal width. However, Figure 12 clearly shows the relationship between the side-chain length and surface viscosity.

Such relationship between the side chain and the viscosity of monolayer is much different from that of the 3-dimensional polymer system like solid bulk polymers and polymer LB films. For instance, the glass transition temperature $\left(T_{\mathrm{g}}\right)$ of solid polymers is generally lowered as the side chain lengthens ${ }^{17}$; which means that the polymer with longer side chains is "softer" at the same temperature. $T_{\mathrm{g}}$ also affects the relaxation behavior of polymer LB films on a solid substrate. The LB films of acetalized PVA begin to exhibit thermal relaxation of 


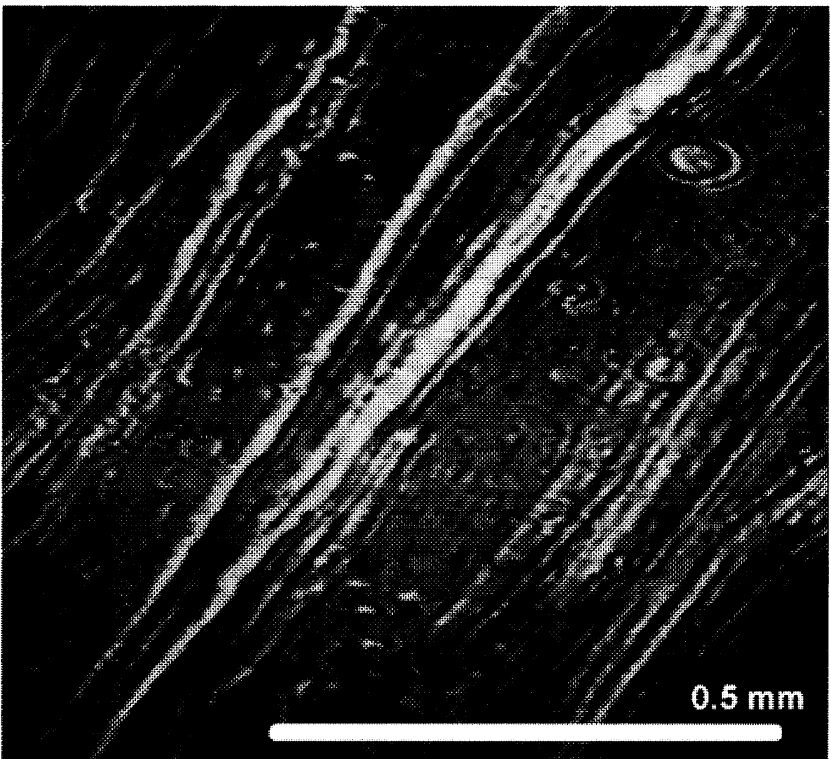

Figure 9. Collapse of a PVD monolayer caused by overcompression $\left(\Pi=34 \mathrm{mN} \mathrm{m}^{-1}\right)$. The compression direction is horizontal in this micrograph.
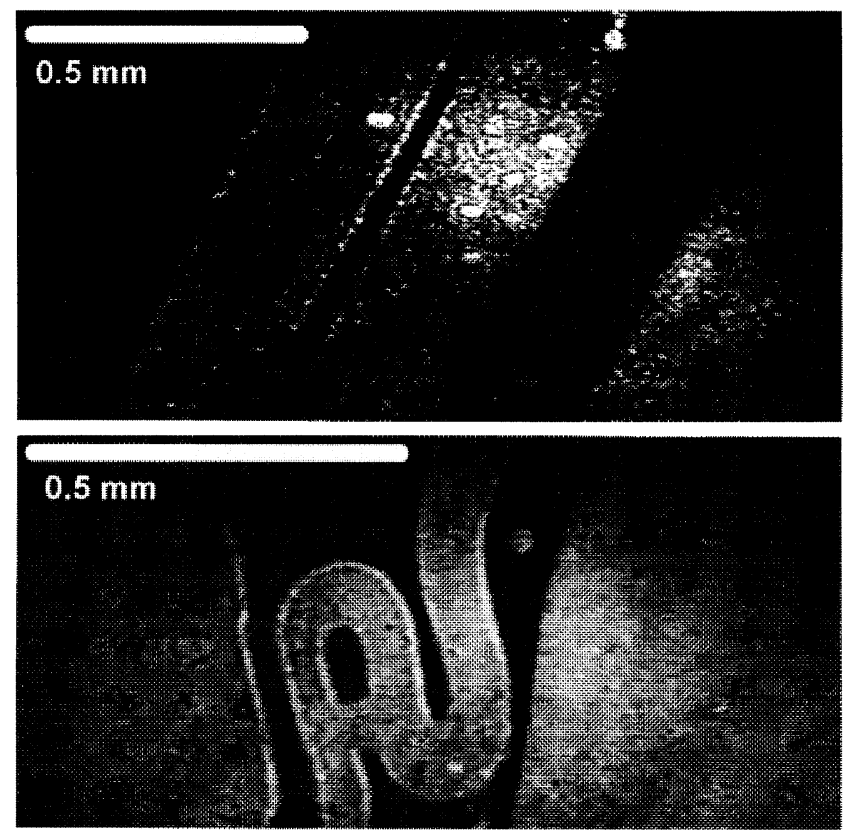

Figure 10. Stripe domain of PVPe (top) and PVO (bottom). Less viscous domains were easily deformed $\left(\Pi=0 \mathrm{mN} \mathrm{m}^{-1}\right)$.

the layer structure at temperature near $T_{\mathrm{g}}$, which was revealed by the excitation energy transfer technique. ${ }^{18}$

The parallel relationship between the length of the side-chain carbons and the viscosity of the monolayer at the air/water interface is attributed to the presence of the water and the effect of the spatial arrangement of the side chains.

In the 3-dimensional system, the interaction between the polymer backbones has a larger effect on the viscoelastic properties compared with the monolayers at the air/water interface. Long side chains reduce the cohesive force among the polymer backbones almost like a plasticizer, which consequently lowers the viscosity and $T_{\mathrm{g}}$ unless the side chains crystallize.

In the case of the polymers forming a monolayer at

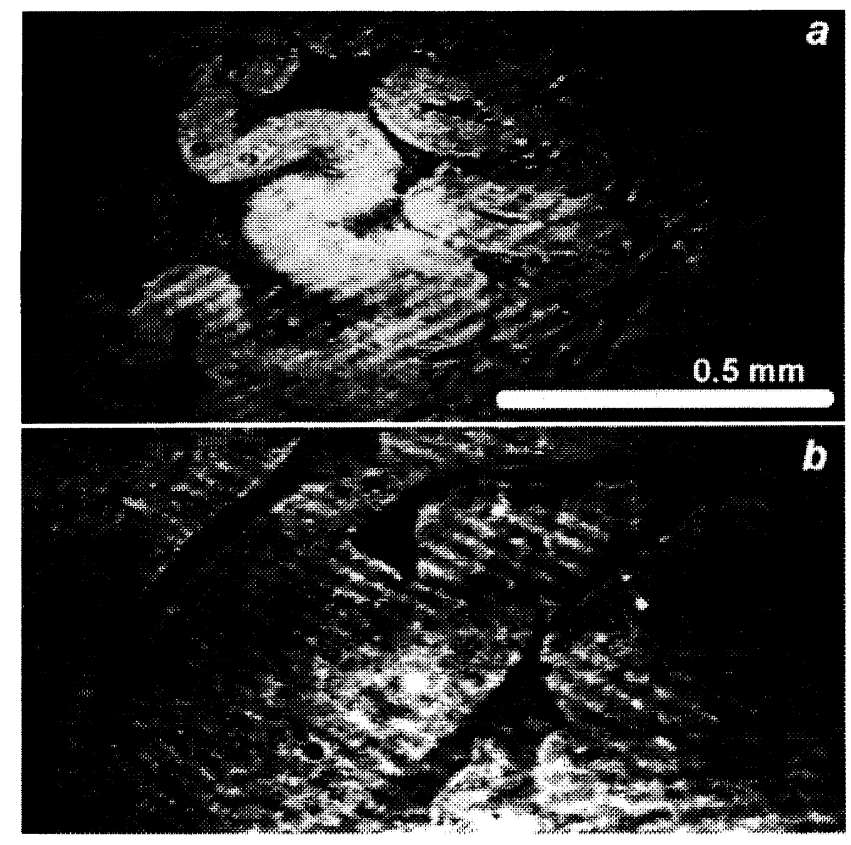

Figure 11. Morphological change of the domains flowing through the canal. Top: (a) PVT domain. A ribbon-like shape remained. Bottom: (b) PVO domain. No characteristic shapes could be observed.

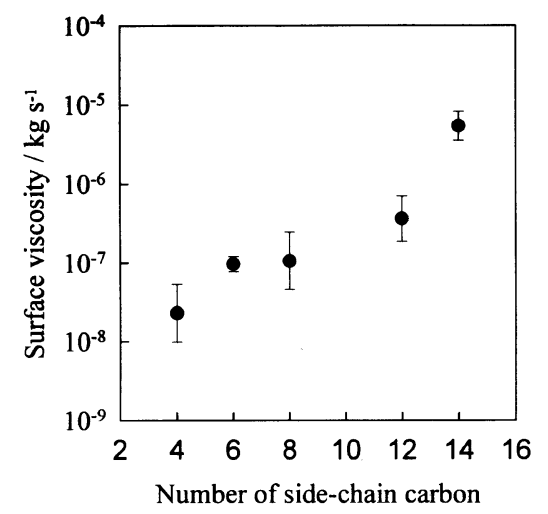

Figure 12. Monolayer viscosity of the acetalized PVAs as a function of side-chain carbon number.

the air/water interface, on the contrary, the side chains interact with one another more effectively because they stick out of the interface and tend to be aligned side by side. The interaction among the polymer backbones is smaller and not affected so much by the alteration of the side chains because the interaction prevails only in the lateral direction owing to the constraint of the polymer backbones on the water surface. By the hydration, the water molecules also reduce the interaction between the polar groups positioned near the polymer backbones. Therefore, the contribution of the cohesive force among side chains exceeds that of the interaction between polymer backbones.

\section{CONCLUSION}

We have demonstrated that the longer side chains of acetalized PVAs raised the viscosity of the monolayers. BAM observation displayed that the monolayers with a shorter side chain were more easily deformed. Canal surface viscometry also demonstrated that the mono- 
layers became more viscous as the side-chain carbons increased in number. These findings show that the cohesive force among side chains plays a greater role in the monolayer viscosity than the 3-dimensional bulk because side chains are aligned protruding out of the interface.

Acetalized PVAs provide homogeneous dispersion of functional groups and good transferability onto a solid substrate. These excellent properties are based on the amorphous and fluid character of the monolayers, arising from a good balance between the cohesive force of the side chains and the flexibility of the main chains on the water surface.

Acknowledgments. This work was supported by a Grant-in-Aid for Scientific Research on Priority Areas, Photochemical Reactions (No. 06239107) from the Ministry of Education, Science, and Culture of Japan. S. I. thanks the Sumitomo Foundation for financial support.

\section{REFERENCES}

1. G. L. Gaines, "Insoluble Monolayers at Liquid-Gas Interfaces,"
Wiley, New York, N.Y., 1966.

2. D. J. Crisp, in "Surface Phenomena in Chemistry and Biology," J. F. Danielli, K. G. A. Pankhurst, and A. C. Riddiford, Ed., Pergamon, London, 1958.

3. A. J. Schouten and G. Wegner, Macromol. Chem., 192, 2203 (1991).

4. T. Nakahara, K. Motomura, and R. Matuura, Bull. Chem. Soc. Jpn., 40, 495 (1967)

5. R. H. G. Brinkhuis and A. J. Schouten, Macromolecules, 24, 1487 (1991).

6. R. H. Tredgold, Thin Solid Films, 152, 223 (1987).

7. K. Naito, J. Colloid Interface Sci., 131, 218 (1989).

8. G. Wegner, Thin Solid Films, 216, 105 (1992).

9. D. J. Crisp, J. Colloid Sci., 1, 49 (1946).

10. D. J. Crisp, J. Colloid Sci., 1, 161 (1946).

11. M. Sacchetti, H. Yu, and G. Zografi, Langmuir, 9, 2168 (1993).

12. M. Watanabe, Y. Kosaka, K. Oguchi, K. Sanui, and N. Ogata, Macromolecules, 21, 2997 (1988).

13. S. Hénon and J. Meunier, Rev. Sci. Instrum., 62, 936 (1991).

14. D. Hönig and D. Möbius, J. Phys. Chem., 95, 4590 (1991).

15. S. Ohmori, S. Ito, and M. Yamamoto, Macromolecules, 23, 4047 (1990)

16. M. Sacchetti, H. Yu, and G. Zografi, Rev. Sci. Instrum., 64, 1941 (1993).

17. K. Oguchi, T. Yoden, K. Sanui, and N. Ogata, Polym. J., 18, 887 (1986).

18. M. Yamamoto, K. Kawano, T. Okuyama, T. Hayashi, and S. Ito, Proc. Jpn. Acad., Ser. B, 70, 121 (1994). 